\title{
Exact solutions of interfacial cracking problem of elliptic inclusion in thermoelectric material
}

\author{
Xin-Kun Du ${ }^{1}$, Ya-Li, Zhang ${ }^{1}$, Sheng-Hu, Ding ${ }^{1 *}$ \\ ${ }^{1}$ School of Mathematics and Statistics, Ningxia University, Yinchuan 750021, China
}

\begin{abstract}
In the present work, the problem for elliptical inclusion with interfacial crack in thermoelectric material is studied. The inclusion and matrix are assumed to be imperfect bonding, which is subjected to uniform heat flux and energy flux at infinity. The interfacial cracking problem of elliptic inclusion in thermoelectric material is investigated by using conformal mapping and complex function method. The complex expressions of temperature field and electric field in inclusion and matrix are obtained. The energy release rate of thermoelectric material under the influence of inclusion is given. The effects of elliptic inclusion with interfacial crack on temperature field and electric potential also are compared by numerical examples. The results show that inclusion reduces the conversion efficiency of thermoelectric material.
\end{abstract}

\section{Introduction}

With the increasing demand for new materials, thermoelectric materials [1-3] are functional materials that can transform thermal energy and electric energy into each other, which has the advantages of simple structure, lightweight, small volume, no moving power components, long life, safety, cleaning and environmental protection. However, many defects are inevitable in the manufacturing and processing of materials, such as microcavity, microcrack, all kinds of inclusions. These defects or second stage materials can also be called inclusions [4-6]. Different from the system with the singled material, composite structures with reinforced phase have more superior behavior. Usually, the composite materials appear with layered and/or inclusion systems, which are common for electric-elastic materials. On the other hand, similar to nearly all the composite materials and structures the mechanical property of thermoelectric materials plays an important role during the manufacture and application process. As a result, many studies forced on the thermoelectric materials [7-11].

Based on conformal mapping function and Mori Tanaka method, Wang [12] studied the effective material properties of elliptical fiber thermoelectric composites. Zhang et al. [13] investigated the two-dimensional problems of an elliptic hole or a rigid inclusion embedded in a thermoelectric material subjected to uniform electric current density and energy flux at infinity based on the complex variable method and conformal mapping technique. Wang et al. [14] analyzed the two-dimensional problem of thermoelectric material with inclined elliptical inclusion and obtained the closed solution of the inclusion problem. In Ref. [15], the problem of three-dimensional ellipsoidal inclusion in thermoelectric material is studied.
By introducing two auxiliary functions, the nonlinear coupled governing equation is successfully transformed into a linear uncoupled equation. Yu et al. [16] studied the plane problem of circular inclusion in thermoelectric medium, and derived the explicit solutions of electric field, temperature field and related thermal stress field in the whole composite. However, as a new intelligent structure, the research on the mechanical properties of thermoelectric materials is still very limited, especially the mechanical properties of inclusions. Important progress has been made in the study of inclusion theory of thermoelectric materials, but the existing results mainly focus on well-bonded inclusions. There are few studies on the non-ideal bonding of inclusion interface such as debonding, cracking and damage. In this paper, the interfacial cracking problem of elliptical inclusion in thermoelectric materials under thermoelectric loadings at infinite distances is studied. Applying the analytical continuation theorem and boundary conditions, then it is transformed into Riemann-Hilbert problem. The complex potential expressions of temperature field and electric field in inclusion and matrix are obtained by conformal transformation and the series expansion.

\footnotetext{
* Corresponding author: dshnx2019@nxu.edu.cn.
} 


\section{Problem formulation}

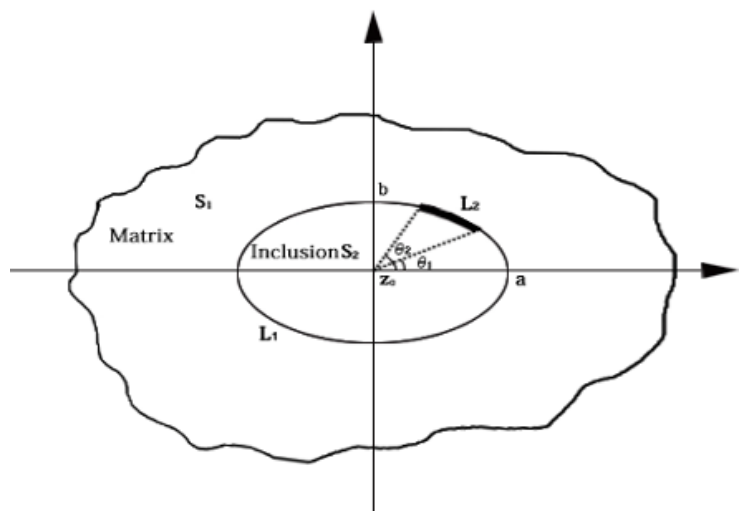

Fig. 1. A schematic diagram of elliptical thermoelectric inclusion with an interfacial crack in an infinite matrix.

The elliptical inclusion embedded in infinite thermoelectric material matrix under far-field thermoelectric load is considered, as shown in Fig. 1. An interfacial crack $L_{2}$ appears at the interface between the inclusion and the matrix due to debonding. Assuming that thermoelectric material is transversely isotropic, the long and short axes of ellipses are divided into $x$ axis and $y$ axis with radii of $a$ and $b$, respectively. In Fig. $1, L_{0}$ represents the interface between inclusion and matrix, $L_{1}$ represents the uncracked part, and $L_{0}=L_{1}+L_{2}$.

The constitutive equations [17]

$$
\begin{gathered}
h=-\kappa \nabla T+s T j \\
j=-\gamma \nabla \varphi-s \gamma \nabla T
\end{gathered}
$$

where $\nabla=\frac{\partial}{\partial x}+\frac{\partial}{\partial y}, h$ is the heat flux along the $x$ and $y$ direction. $j$ is energy flux. $T$ is temperature, $\varphi$ is electric potential, $\kappa$ is thermal conductivity, $s$ is seebeck's coefficient, $\gamma$ is electric conductivity and $\beta=s T$ is Peltier coefficient.

From Eqs. (1) and (2), we can get

$$
\begin{aligned}
& (\gamma s \beta+\kappa) \nabla^{2} T+\gamma \beta \nabla^{2} \varphi=0 \\
& \gamma s \nabla^{2} T+\gamma \nabla^{2} \varphi=0
\end{aligned}
$$

where $\nabla^{2}$ is the two-dimensional Laplace operator.

From Eq. (3), we can take the real part of the analytic function as follows

$$
\begin{aligned}
& \mathrm{T}=\frac{1}{2}[\Psi(z)+\overline{\Psi(z)}] \\
& \varphi=\frac{1}{2}[\Phi(z)+\overline{\Phi(z)}]
\end{aligned}
$$

where $z=x+i y, \Psi(z)$ and $\Phi(z)$ are analytic functions, and '-, means complex conjugate.

The interface conditions of matrix and inclusion can be expressed as

$$
\begin{array}{lll}
h_{n}^{(1)}=h_{n}^{(2)}=0 & j_{n}^{(1)}=j_{n}^{(2)}=0 & \text { on } L_{2}(5) \\
\mathrm{T}^{(1)}=\mathrm{T}^{(2)}=0 & \varphi^{(1)}=\varphi^{(2)}=0 & \text { onL }_{1}(6)
\end{array}
$$

where the superscript (1) and (2) represent the matrix and inclusion region respectively. Here $n$ is the normal direction of the interface.

Now, introduce the following mapping function

$$
\begin{aligned}
& z=\Psi(\zeta)=R\left(\zeta+m \zeta^{-1}\right) \\
& \zeta=\left(z+\sqrt{z^{2}-c^{2}}\right) /(2 R)
\end{aligned}
$$

where $R=\frac{a+b}{2}, m=\frac{a-b}{a+b}, c=\sqrt{a^{2}-b^{2}}$.
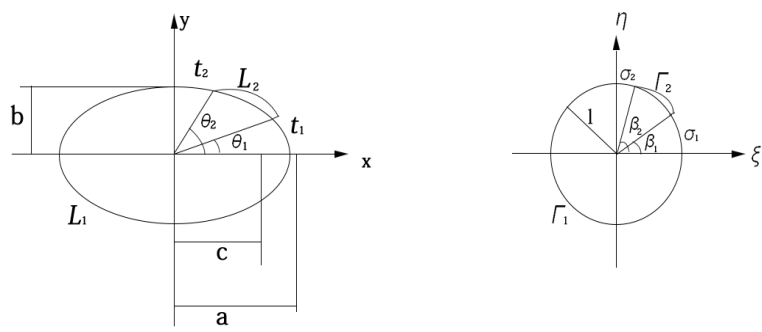

Fig. 2. The conformal transformation

Under the above transformation, the interface conditions (5) and (6) can be expressed as

$\sigma \Psi^{\prime}(\sigma)+\overline{\sigma^{\prime}(\sigma)}=0$,

$\sigma \Phi^{\prime}(\sigma)+\overline{\sigma \Phi^{\prime}(\sigma)}=0 \quad \sigma \in \Gamma_{2}$

$\sigma \Psi^{\prime}(\sigma)-\overline{\sigma \Psi^{\prime}(\sigma)}=0$,

$\sigma \Phi^{\prime}(\sigma)-\overline{\sigma \Phi^{\prime}(\sigma)}=0 \quad \sigma \in \Gamma_{1}$

\section{Theoretical solution of the problem}

Introducing the new complex potential functions

$$
\begin{aligned}
& u(\zeta)=\zeta \Psi^{\prime}(\zeta), \\
& v(\zeta)=\zeta \Phi^{\prime}(\zeta) \quad|\zeta|>1
\end{aligned}
$$

By analytically extending the complex function defined above in the matrix region to the inclusion region, one has

$$
\begin{aligned}
& u(\zeta)=-\frac{1}{\zeta} \overline{\Psi^{\prime}}\left(\frac{1}{\zeta}\right), \\
& v(\zeta)=-\frac{1}{\zeta} \overline{\Phi^{\prime}}\left(\frac{1}{\zeta}\right) \quad 0<|\zeta|<1
\end{aligned}
$$

In this way, the interface conditions (8) and (9) can be reduced to Riemann-Hilbert problem as

$$
\begin{array}{ll}
u^{+}(\sigma)-u^{-}(\sigma)=0, & \sigma \in \Gamma_{2} \\
u^{+}(\sigma)+u^{-}(\sigma)=0, & \sigma \in \Gamma_{1} \\
v^{+}(\sigma)-v^{-}(\sigma)=0, & \sigma \in \Gamma_{2} \\
v^{+}(\sigma)+v^{-}(\sigma)=0, & \sigma \in \Gamma_{1}
\end{array}
$$

The solution of equations (12)-(15) can be expressed as 


$$
\begin{aligned}
& u(\zeta)=F(\zeta)\left(S^{*}+\sum_{n=0}^{\infty} l_{n} \zeta^{n+1}+\sum_{n=0}^{\infty} S_{n} \zeta^{-(n+1)}\right) \\
& v(\zeta)=F(\zeta)\left(\overline{S^{*}}+\sum_{n=0}^{\infty} \bar{l}_{n} \zeta^{n+1}+\sum_{n=0}^{\infty} \overline{S_{n}} \zeta^{-(n+1)}\right)
\end{aligned}
$$

In the formula $F(\zeta)$ is a Plemelj function and the expression is

$$
F(\zeta)=\left[\left(\zeta-\sigma_{1}\right)\left(\zeta-\sigma_{2}\right)\right]^{-1 / 2}
$$

The analytic functions $\Psi(\zeta)$ and $\Phi(\zeta)$ can be expanded in the matrix region as Laurent series

$$
\begin{aligned}
& \Psi(\zeta)=\sum_{n=0}^{\infty}\left(a_{n} \zeta^{n+1}+b_{n} \zeta^{-(n+1)}\right) \\
& \Phi(\zeta)=\sum_{n=0}^{\infty}\left(c_{n} \zeta^{n+1}+d_{n} \zeta^{-(n+1)}\right) \quad|\zeta|>1
\end{aligned}
$$

where

$$
\begin{aligned}
& b_{0}^{(2)}=-\left(\alpha_{0}^{*} S^{*}+\sum_{m=0}^{\infty} \alpha_{m+1}^{*} l_{m}\right) \\
& d_{0}^{(2)}=-\left(\alpha_{0}^{*} \overline{S_{*}}+\sum_{m=0}^{\infty} \alpha_{m+1}^{*} \overline{l_{m}}\right) \\
& b_{n}^{(2)}=-\frac{1}{n+1}\left(\alpha_{n}^{*} S^{*}+\sum_{m=0}^{\infty} \alpha_{n+m+1}^{*} l_{m}+\sum_{m=0}^{n-1} a_{m}^{*} S_{n-m-1}\right)(n>0) \\
& d_{n}^{(2)}=-\frac{1}{n+1}\left(\alpha_{n}^{*} \overline{S^{*}}+\sum_{m=0}^{\infty} \alpha_{n+m+1}^{*} \overline{l_{m}}+\sum_{m=0}^{n-1} a_{m}^{*} \overline{S_{\mathrm{n}-m-1}^{*}}\right)(n>0)
\end{aligned}
$$

with

$$
\begin{aligned}
& \alpha_{n}^{*}=\sum_{m=0}^{n} \gamma_{m}^{*} \gamma_{n-m}^{*} \sigma_{1}^{m} \sigma_{2}^{n-m}, \quad \gamma_{m}^{*}=\frac{(2 m) !}{2^{2 m}(m !)^{2}} \\
& \beta_{\mathrm{n}}=\sum_{m=0}^{n} \gamma_{m} \gamma_{n-m} \sigma_{1}^{-m} \sigma_{2}^{-(n-m)} \\
& \beta_{n}^{*}=\sum_{m=0}^{n} \gamma_{m}^{*} \gamma_{n-m}^{*} \sigma_{1}^{-m} \sigma_{2}^{-(n-m)} \\
& S^{*}=-\frac{1}{F(0)} \sum_{m=0}^{\infty}(m+1) \beta_{m+1} \overline{a_{m}^{(2)}} \\
& \overline{S^{*}}=-\frac{1}{F(0)} \sum_{m=0}^{\infty}(m+1) \beta_{m+1} \overline{c_{m}^{(2)}} \\
& l_{0}=\sum_{m=0}^{\infty}(m+1) \alpha_{m+1} \alpha_{m}^{(2)} \\
& \overline{l_{0}}=\sum_{m=0}^{\infty}(m+1) \alpha_{m+1} c_{m}^{(2)} \\
& l_{n}=\sum_{m=0}^{\infty}(m+n) \alpha_{m} a_{m+n-1}^{(2)}, \\
& \overline{l_{n}}=\sum_{m=0}^{\infty}(m+n) \alpha_{m} c_{m+n-1}^{(2)} \quad(k \geq 1)
\end{aligned}
$$

$S_{n}=-\frac{1}{F(0)} \sum_{m=0}^{\infty}(m+n+1) \beta_{m} \overline{a_{m+n}^{(2)}}$,

$\overline{S_{n}}=-\frac{1}{F(0)} \sum_{m=0}^{\infty}(m+n+1) \beta_{m} \overline{c_{m+n}^{(2)}} \quad(k \geq 0)$

As an example, we consider the case where the interfacial cracks are symmetrically distributed relative to the $x$ axis. When the far field only acts on that uniform heat flux $h_{x}^{\infty}$ and $h_{y}^{\infty}$ the electric flux loadings are impermeable, one gets

$$
a_{0}^{(2)}=\frac{1}{2} a(1+\varepsilon)\left(h_{x}^{\infty}-h_{y}^{\infty}\right)\left[M^{(2)}\right]^{-1}, \quad a_{n}^{(2)}=0 \quad(n \geq 1)
$$

$c_{0}^{(2)}=-\frac{1}{2} a(1+\varepsilon)\left(h_{x}^{\infty}-h_{y}^{\infty}\right)\left[M^{(2)}\right]^{-1}, \quad c_{n}^{(2)}=0 \quad(n \geq 1)$

where $M$ is the material constant matrix which can be expressed as follows $M=\left[\begin{array}{cc}-(\gamma s \beta+\kappa) & -\gamma \beta \\ -\gamma S & -\gamma\end{array}\right]$.

Since energy flux $j$ is transported by both electric current density $e$ and heat flux $h$, thus energy flux $j$ can be expressed as

$$
j=h+e v
$$

Defining the current intensity factor $K_{e}$, heat flux intensity factor $K_{h}$ and energy flux intensity factor $K_{j}$, one obtains

$$
\begin{gathered}
K_{e}=\lim _{\theta \rightarrow \alpha} \sqrt{2 \pi(\theta-\alpha)} e \\
K_{h}=\lim _{\theta \rightarrow \alpha} \sqrt{2 \pi(\theta-\alpha)} h \\
K_{j}=\lim _{\theta \rightarrow \alpha} \sqrt{2 \pi(\theta-\alpha)} j
\end{gathered}
$$

where $\alpha=\beta_{2}-\beta_{1}, K_{e 0}=\sqrt{2 \pi \alpha} e^{\infty}$,

$$
K_{h 0}=\sqrt{2 \pi \alpha} h^{\infty} \text {, and } K_{j 0}=\sqrt{2 \pi \alpha} j^{\infty} \text {. }
$$

\section{Numerical analysis}

In this section, the influence of the inclusion shape on the thermoelectric field and temperature will be analyzed. Fig. 3 shows the change of temperature with the interface crack angle under different $a / b$ values. It can be seen that the temperature decreases with the increase of the interface crack angle, which shows that the interface crack reduces the properties of thermoelectric materials 


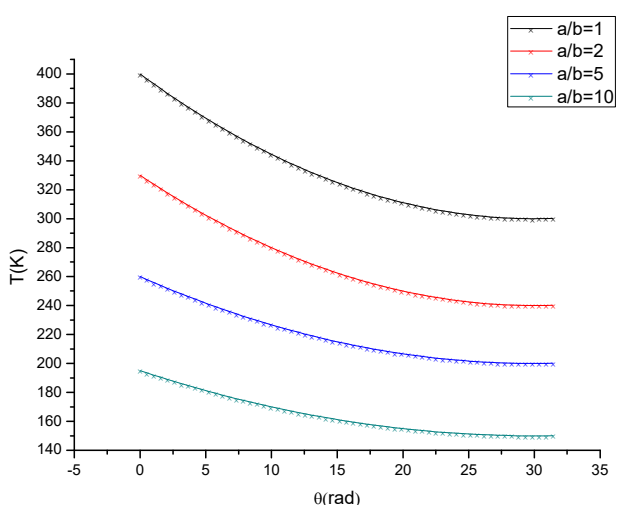

Fig. 3. The temperature changes with the crack angle of the interface at different $\mathrm{a} / \mathrm{b}$ values

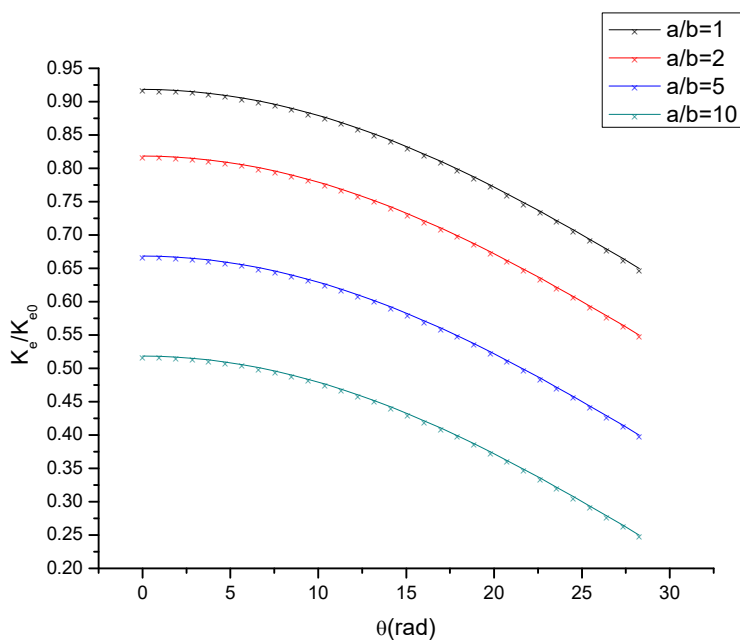

Fig. 4. The current intensity factor changes with the crack angle of the interface at different $\mathrm{a} / \mathrm{b}$ values

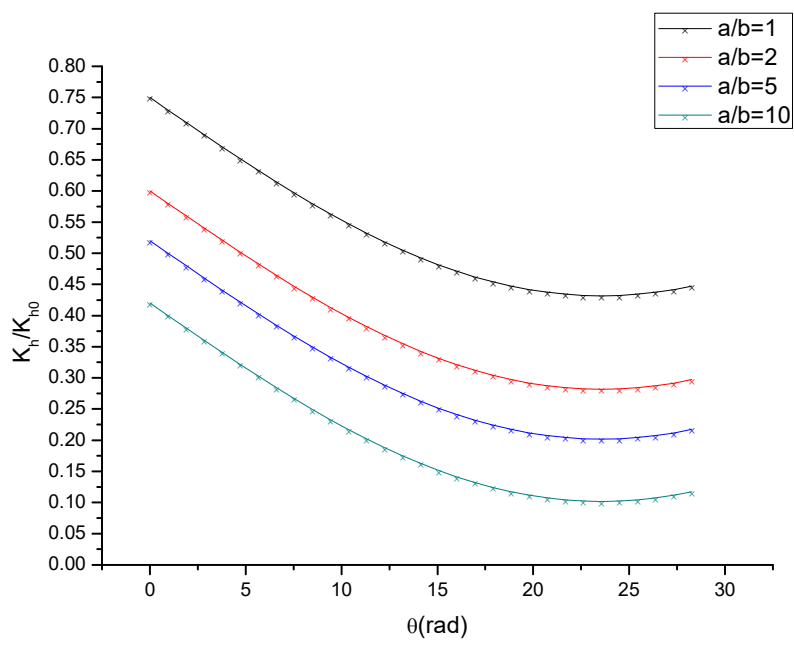

Fig. 5. The heat flux intensity factor changes with the crack angle of the interface at different $\mathrm{a} / \mathrm{b}$ values

Figs.4-5 show the change of dimensionless current intensity factor and heat flux intensity factor with the interface crack angle under different $\mathrm{a} / \mathrm{b}$ values. It can be found that two physical quantities decrease with the increase of the interface crack angle, which indicates that the interface crack reduces the conversion efficiency of thermoelectric materials.

\section{Conclusion}

In this paper, using the conforming mapping and the theorem of analytic continuation, the thermoelectric medium with elliptical inclusion of interfacial cracking problem is studied. Based on the complex variable method and series expansion, exact solutions are presented in the matrix and inclusion. From the obtained expressions, it can be found that the thermoelectric field and electric potential depend on the material constants of each phase, the geometric parameters of the system and the conditions of the thermoelectric loadings at infinity. Numerical examples are given to discuss the influence of inclusion size, inclusion volume fraction and inclusion properties. The result shows that the inclusion reduces the conversion efficiency of thermoelectric materials. In the case of multiinclusion interaction, it is not enough to use only the inclusion density parameters. The inclusion distributions should be considered and extracted.

\section{Acknowledgement}

The research was supported by the National Natural Science Foundation of China (11762016, 11762017, 12062021 and 12062022) and the Natural Science Foundation of Ningxia (2020AAC03057) are gratefully acknowledged.

\section{References}

1. F. J. Disalvo, Science, 285(5428): 703-707, (1999)

2. G. Snyder, E. Toberer, Nat Mater, 7(2):105-114, (2008)

3. M. S. Dresselhaus, G. Chen, M. Y. Tang, R. G. Yang, H. Lee, D. Z. Wang, Z. F. Ren, J.P. Fleurial, P. Gogna, Adv Mater, 19(8): 1043-1053, (2010)

4. Y. Huang, K. X. Hu, A. Chandra, J. Appl. Mech, 62(1): 71-77, (1995)

5. A. Mattoni, L. Colombo, F. Cleri, Phys. Rev. B, 70(70): 094108, (2004)

6. V. Zlatic, A. C. Hewson, Springer Netherlands: 340(2009)

7. H. P. Song, C. F. Gao, J. Y. Li, J. Therm. Stresses, 38(3): 325-337, (2015)

8. C. B. Yu, D. F. Zou, Y. H. Li, H. B. Yang, C. F. Gao, Acta. Mech, (2):1-20(2017)

9. S. H. Ding, Y.T. Zhou, Acta Mech. Solida Sin, 4(5): 12-522(2018)

10. S. H. Ding, Q. N. Liu, CMES-Comp. Model. Eng. Sci, 114(3): 335-349(2018)

11. Y. J. Cui, K. F. Wang, B. L. Wang, Appl. Math. Mech.-Engl. Ed: 1-8 (2018)

12. Y. Z. Wang, Appl. Phys. A-Mater. Sci. Process, 119(3): 1081-1085 (2015)

13. A. B. Zhang, B. L. Wang, J. Wang, Int. J. Therm. Sci, 117: 184-19(2017) 
14. P. Wang, B. L.Wang, K. F.Wang, Y. J.Cui, Compos. Pt. B-Eng, 166: 130-138 (2019)

15. P. Wang, B. L.Wang, K. F. Wang, H. Hirakata, C. Zhang, Int. J. Eng. Sci, 142(SEP.): 158-169(2019)
16. C. B. Yu, H. B. Yang, Y. H. Li, K. S, C. F. G, Z. Angew, Math. Mech, 99(8): e201800240(2019)

17. A. B. Zhang, B. L. Wang. Theor. Appl. Fract. Mech, 66(16): 33-36(2013) 